\title{
Validation of the Korean Version of the Delirium Motor Subtype Scale
}

\author{
Hyunkyung Kim¹, Miji Lee ${ }^{1}$, In Sun Kwon², and Jeong Lan Kim ${ }^{1,3 凶}$ \\ ${ }^{1}$ Department of Psychiatry, Chungnam National University Hospital, Daejeon, Republic of Korea \\ ${ }^{2}$ Chungnam National University Hospital Clinical Trials Center, Daejeon, Republic of Korea \\ ${ }^{3}$ Department of Psychiatry, School of Medicine, Chungnam National University, Daejeon, Republic of Korea
}

\begin{abstract}
Objective The Delirium Motor Subtype Scale (DMSS) is a validated and reliable instrument developed from various methods previously used to assess delirium motor subtypes. It focuses on pure motor disturbances with a relative specificity for delirium. The aim of this study was to investigate the validity and reliability of a Korean version of the DMSS (K-DMSS).
\end{abstract}

Methods We recruited 145 patients who were older than 60 years and had been referred for psychiatric consultation for delirium. These patients were evaluated using the K-DMSS, Liptzin \& Levkoff criteria, and the Korean version of the Delirium Rating Scale-Revised-98 (K-DRS-R98) to compare delirium motor subtypes.

Results The internal consistency of the K-DMSS in assessing delirium motor subtypes was acceptable (Cronbach's alpha=0.79). Delirium motor subtypes identified with the K-DMSS and K-DRS-R98 showed almost perfect agreement (Cohen's Kappa=0.81), while those identified with the K-DMSS and Liptzin \& Levkoff criteria showed substantial agreement (Cohen's Kappa=0.78).

Conclusion Our results suggest that the K-DMSS is a valid and reliable tool for identifying delirium subtypes.

Psychiatry Investig 2018;15(1):6-12

Key Words Delirium, Motor, Subtypes, DMSS.

\section{INTRODUCTION}

The assessment and identification of delirium motor subtypes is clinically important because these subtypes may differ in terms of the severity of symptoms, ${ }^{1}$ etiology, ${ }^{2}$ pathophysiology, ${ }^{3}$ and outcome. ${ }^{4,5}$ In addition, delirium is misdiagnosed or detected late in half to two-thirds of cases, which leads to delayed treatment. ${ }^{6}$ Delirium is likely to be misdiagnosed as psychosis, mania, or akathisia in patients with the hyperactive or mixed subtypes, and as depression or dementia in patients with the hypoactive subtype. ${ }^{5}$

Altered motor behavior in patients with cognitive disturbance has long been recognized, with the ancient Greeks de-

Received: April 27, 2017 Revised: August 3, 2017

Accepted: September 11, 2017 Available online: October 17, 2017

$\bowtie$ Correspondence: Jeong Lan Kim, MD, PhD

Department of Psychiatry, Chungnam National University Hospital, 282 Munhwa-ro, Jung-gu, Daejeon 35015, Republic of Korea

Tel: +82-42-280-7287, Fax: +82-42-280-7886, E-mail: kimjl@cnu.ac.kr

(a) This is an Open Access article distributed under the terms of the Creative Commons Attribution Non-Commercial License (http://creativecommons.org/licenses/by$\mathrm{nc} / 4.0$ ) which permits unrestricted non-commercial use, distribution, and reproduction in any medium, provided the original work is properly cited. scribing "agitated phrenitis" and "lethargicus"? Since the introduction of terms such as "hyperactive", "hypoactive", and "mixed" in the contemporary literature, ${ }^{8,9}$ there have been new approaches to define motor subtypes using different methods. ${ }^{40-12}$ However, attempts to categorize the subtype have produced heterogeneous results due to differences in the populations assessed and the methods used to define motor subtypes. In a more recent study, commonly used methods ${ }^{9-11,13}$ were compared and the authors reported just 34\% concordance between the methods within the same population. ${ }^{14}$

To develop a valid instrument to assess motor subtypes of delirium, the Delirium Motoric Checklist (DMC) ${ }^{14}$ was developed by combining 30 features from three existing psychomotor check lists. ${ }^{9-11}$ Among these 30 features, 11 were identified as specific to patients with delirium and correlated with the Delirium Rating Scale-Revised-98 (DRS-R98), ${ }^{13}$ which resulted in a new motor subtype scale called the Delirium Motor Subtype Scale (DMSS). ${ }^{15}$ The DMSS focuses on "pure" motor symptoms rather than psychomotoric symptoms. Other studies have shown that the DMSS has good concurrent and predictive validity compared with objective methods for mea- 
suring motor activity, including electronic methods such as accelerometry. ${ }^{16}$ In addition, the scale is simple enough for use by both medical and non-medical staff.

In Korea, there has been no study of the DMSS, although some authors have investigated the associations between the delirium motor subtype and the severity of delirium and clinical characteristics. ${ }^{17}$

Therefore, the aim of this study was to develop a Korean version of the DMSS (K-DMSS) and investigate the validity and reliability of this scale among elderly patients with delirium.

\section{METHODS}

\section{Subjects}

In this study, we recruited 145 elderly patients with delirium diagnosed according to DSM-5 criteria. ${ }^{18}$ All patients had been admitted to a university hospital in South Korea, and subsequently referred to the psychiatric consultation liaison service from any medical or surgical ward. Patients with history of dementia, schizophrenia, bipolar disorder and other severe psychiatric disorder were excluded. We excluded patients suspected to have dementia on the basis of the information provided by caregivers according to DSM- 5 criteria. ${ }^{18}$ We also excluded patients who were admitted to the intensive care unit or emergency room and who refused to participate.

The procedures and rationale for the study, as well as the right to withdraw, were explained to all the patients and their caregivers. Due to the nature of the study, informed consent was obtained from patients where possible, otherwise proxy consent was obtained for caregivers. The study protocol was approved by the Institutional Review Board (IRB No. 2015-04-009).

\section{Assessment tools}

\section{K-DMSS}

The DMSS is an 11-item scale used to classify delirium into several motoric subtypes. ${ }^{15}$ Each item is rated as "absent (score= 0)" or "present (score=1)" on the basis of the patient's status over the previous $24 \mathrm{~h}$. The DMSS hyperactive subtype is assigned if the patient shows at least two of the following symptoms: 1) increased quantity of motor activity; 2) loss of control of motor activity; 3) restlessness; and 4) wandering. The hypoactive subtype is assigned if the patient shows at least two of the following symptoms: 1) decreased amount of activity;2) decreased speed of actions; 3) reduced awareness of surroundings; 4) decreased amount of speech; 5) decreased speed of speech; 6) listlessness; and 7) reduced alertness/withdrawal. The mixed subtype is assigned if the patient shows both hyperactive and hypoactive symptoms. No motor subtype is assigned if there is no evidence of either the hyperactive or hypoactive subtype.
To develop the K-DMSS, the DMSS was first translated into Korean by two psychiatrists after obtaining approval by David Meagher, one of the original developers of the DMSS. A bilingual individual conducted back translation and the translation was subsequently reviewed by other psychiatrists in our study team, as well as an English speaker.

\section{K-DRS-R98}

The K-DRS-R98 is a 16-item scale comprising 13 items for severity and 3 items for diagnosis. It was validated and standardized in Korean by Lim et al. ${ }^{19}$ Each item is rated from 0 (absent) to 2 or 3 (severe), with a maximum severity score of 39 and total scale score of 46 . Among the severity items, items \#7 and \#8 rate motor agitation and motor retardation, respectively. For classification of the delirium motor subtype, the hyperactive subtype is assigned if the score for item \#7 ranges from 1 to 3 with a score for item \#8 of 0 . Meanwhile, the hypoactive subtype is assigned if the score for item $\# 8$ ranges from 1 to 3 with a score for item \#7 of 0 . The mixed subtype is assigned if the scores both these items range from 1 to 3 , and no motor subtype is assigned if both scores are 0 .

\section{Liptzin \& Levkoff criteria}

Liptzin \& Levkoff criteria ${ }^{10}$ are adapted from the Delirium Symptom Interview (DSI), ${ }^{20}$ and have been applied to categorize the delirium motor subtype and to compare between methods. The hyperactive subtype is assigned if a patient has three or more of the following symptoms: hypervigilance, restlessness, fast or loud speech, irritability, combativeness, impatience, swearing, singing, laughing, uncooperativeness, euphoria, anger, wondering, easy startling, fast motor responses, distractibility, tangentiality, nightmares, and persistent thoughts. The hypoactive subtype is assigned if a patient has four or more of the following symptoms: unawareness, decreased alertness, sparse or slow speech, lethargy, slowed movements, staring, and apathy. If they meet criteria for both motor subtypes, the mixed subtype is assigned.

\section{Study procedures}

This was a prospective study in which medical information was obtained from patients, physicians, nurses, caregivers, and medical records. A trained psychiatrist assessed patients with the K-DMSS, while another trained psychiatrist independently assessed patients with the K-DRS-R98 and Liptzin \& Levkoff criteria. In a subset of patients, the K-DMSS was conducted a third psychiatrist to investigate inter-rater reliability.

\section{Statistical analysis}

We used Cronbach's alpha to assess the internal consistency of the K-DMSS. We used Cohen's kappa statistic, sensitiv- 
ity, specificity, and correspondence analysis to compare each motor subtype as defined by the K-DMSS with each motor subtype as defined by the K-DRS-R98 and Liptzin \& Levkoff criteria. To investigate inter-rater reliability, we used Cohen's kappa statistic. Cohen's kappa coefficients were interpreted according to the guidelines proposed by Landis and Koch: 'almost perfect' (0.81-1); 'substantial' (0.61-0.80); 'moderate' (0.41-0.60); 'fair' (0.21-0.40); 'slight' (0.00-0.20); or 'poor' $(<0.00)$ agreement. ${ }^{21}$ All statistical analyses were conducted using SPSS version 22.0 (IBM Corp., Armonk, NY, USA) and statistical significance was accepted at $\mathrm{p}<0.05$.

\section{RESULTS}

\section{Patient demographic and clinical characteristics}

We included 145 patients (mean age, $75.97 \pm 7.09$ years) with delirium in the present study. Of these, 87 were male (mean age, $74.54 \pm 7.30$ years) and 58 were female (mean age, 78.12 \pm 6.24 years). Participants had mean K-DRS-R98 total and severity scores of $28.10 \pm 5.89$ and $21.95 \pm 5.59$, respectively. The most common primary etiology of the delirium was infection (systemic), followed by other, neoplasm (systemic), and organic insufficiency (Table 1), according to the Delirium Etiology Checklist (DEC) classification. ${ }^{22}$

\section{Delirium motor subtype distribution}

Each patient was classified into the hyperactive subtype, hypoactive subtype, mixed subtype, or no motor subtype according to the K-DMSS, K-DRS-R98 and Liptzin \& Levkoff criteria (Figure 1). According to the K-DMSS, the most common motor subtype was the hyperactive subtype $(n=72$, $50 \%)$, followed by the mixed subtype ( $n=60,41 \%)$, hypoactive subtype $(n=9,6 \%)$, and no motor subtype $(n=4,3 \%)$.

Table 1. Primary etiologies underlying delirium in patients $(\mathrm{N}=145)$

\begin{tabular}{lc}
\hline \multicolumn{1}{c}{ Etiologies } & $\mathrm{N}$ \\
\hline Drug intoxication & 0 \\
Drug withdrawal & 1 \\
Metabolic/Endocrine disturbance & 2 \\
Traumatic brain injury & 5 \\
Seizures & 0 \\
Infection (intracranial) & 1 \\
Infection (systemic) & 36 \\
Neoplasm (intracranial) & 2 \\
Neoplasm (systemic) & 29 \\
Cerebrovascular & 9 \\
Organ insufficiency & 25 \\
Other CNS & 0 \\
Other & 35 \\
\hline
\end{tabular}

According to the K-DRS-R98, the most common subtype was the mixed subtype ( $n=70,48 \%)$, followed by the hyperactive subtype $(n=64,44 \%)$, hypoactive subtype $(n=9,6 \%)$, and no motor subtype $(n=2,1 \%)$. According to the Liptzin \& Levkoff criteria, the hyperactive subtype $(n=80,55 \%)$ is most common, followed by the mixed subtype ( $n=47,32 \%)$, hypoactive subtype $(n=10,7 \%)$, and no motor subtype $(n=8,6 \%)$.

\section{Reliability}

The internal consistency of the K-DMSS was assessed with Cronbach's alpha coefficient (Table 2), with a coefficient for the total items of 0.79 . As each item was removed, the internal consistency persisted, with Cronbach's alpha ranged from 0.74 to 0.82 .

For 50 patients, the K-DMSS was assessed by two psychiatrists independently during the same interview to investigate the inter-rater reliability. There was almost perfect agreement, with Cohen's kappa coefficient of $0.86(\mathrm{p}<0.001)$.

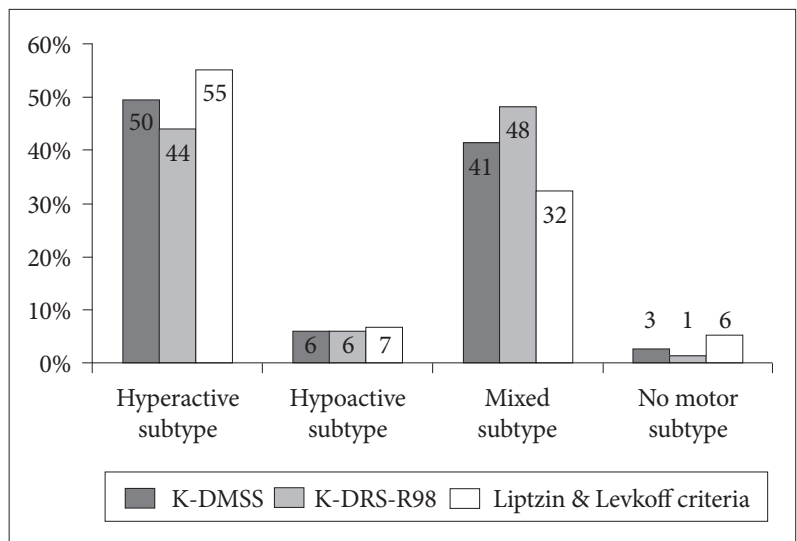

Figure 1. Number of delirious patients within each motor subtype.

Table 2. Internal consistency of K-DMSS

\begin{tabular}{lc}
\hline & Cronbach's alpha \\
\hline Total & 0.79 \\
\hline Items & If item deleted \\
\hline Increased quantity of motor activity & 0.81 \\
Loss of control of activity & 0.81 \\
Restlessness & 0.82 \\
Wandering & 0.82 \\
Decreased amount of activity & 0.74 \\
Decreased speed of actions & 0.75 \\
Reduced awareness of surroundings & 0.75 \\
Decreased amount of speech & 0.75 \\
Decreased speed of speech & 0.75 \\
Listlessness & 0.76 \\
Reduced alertness/withdrawal & 0.76 \\
\hline
\end{tabular}


Table 3. Number of the patients within each motor subtype based on K-DMSS and K-DRS-R98 ( $N=145)$

\begin{tabular}{lcccrr}
\hline \multirow{2}{*}{ K-DMSS motor subtype } & \multicolumn{3}{c}{ K-DRS-R98 motor subtype } \\
\cline { 2 - 6 } & Hyperactive & Hypoactive & Mixed & No & Total \\
\hline Hyperactive & 61 & 0 & 11 & 0 & 72 \\
Hypoactive & 0 & 8 & 1 & 0 & 9 \\
Mixed & 1 & 1 & 58 & 0 & 60 \\
No & 2 & 0 & 70 & 2 & 145 \\
Total & 64 & 9 & 0 & 2 & 145 \\
\hline
\end{tabular}

Table 4. Agreement, sensitivity and specificity between K-DMSS and K-DRS-R98 $(\mathrm{N}=145)$

\begin{tabular}{lccc}
\hline \multicolumn{1}{c}{ K-DMSS motor subtype } & Cohen's $\kappa$ & Sensitivity (CI) & Specificity (CI) \\
\hline Hyperactive subtype & 0.81 & $0.95(0.86-0.99)$ & $0.86(0.77-0.93)$ \\
Hypoactive subtype & 0.88 & $0.89(0.51-0.99)$ & $0.99(0.95-1.00)$ \\
Mixed subtype & 0.81 & $0.83(0.72-0.90)$ & $0.97(0.90-1.00)$ \\
No motor subtype & 0.66 & $1(0.20-1.00)$ & $0.99(0.95-1.00)$ \\
\hline
\end{tabular}

Overall Cohen's $\kappa=0.81$. CI: confidence interval

Table 5. Number of the patients within each motor subtype based on K-DMSS and Liptzin \& Levkoff criteria $(\mathrm{N}=145)$

\begin{tabular}{lccccc}
\hline \multirow{2}{*}{ K-DMSS motor subtype } & \multicolumn{4}{c}{ Liptzin \& levkoff criteria motor subtype } \\
\cline { 2 - 6 } & Hyperactive & Hypoactive & Mixed & No & Total \\
\hline Hyperactive & 68 & 0 & 1 & 3 & 72 \\
Hypoactive & 1 & 8 & 0 & 0 & 9 \\
Mixed & 11 & 2 & 0 & 1 & 4 \\
No & 0 & 0 & 47 & 8 & 4 \\
Total & 80 & 10 & 46 & 145 \\
\hline
\end{tabular}

Table 6. Agreement, sensitivity and specificity between K-DMSS and Liptzin \& Levkoff criteria (N=145)

\begin{tabular}{lccr}
\hline \multicolumn{1}{c}{ K-DMSS motor subtype } & Cohen's $\kappa$ & Sensitivity (CI) & Specificity (CI) \\
\hline Hyperactive subtype & 0.78 & $0.85(0.75-0.92)$ & $0.94(0.84-0.98)$ \\
Hypoactive subtype & 0.83 & $0.80(0.44-0.96)$ & $0.99(0.95-1.00)$ \\
Mixed subtype & 0.78 & $0.98(0.87-1.00)$ & $0.86(0.77-0.92)$ \\
No motor subtype & 0.65 & $0.5(0.17-0.83)$ & $1(0.97-1.00)$ \\
\hline
\end{tabular}

Overall Cohen's $\kappa=0.78$. CI: confidence interval

\section{Concurrent validity}

The agreement of subtype classification between the KDMSS and K-DRS-R98 (Tables 3 and 4) was almost perfect, with a Cohen's kappa coefficient of $0.81(\mathrm{p}<0.001)$. Table 4 shows the additional analyses for the agreement, sensitivity, and specificity for each subtype. When the patients were classified with the K-DMSS and Liptzin \& Levkoff criteria (Tables 5 and 6), Cohen's kappa was $0.78(\mathrm{p}<0.001)$, and Table 6 shows the further analyses for each subtype.

Correspondence analysis, which graphically represents the relationships between the same subtypes categorized by each scale, showed that the subtypes did not differ from each other (Figures 2 and 3).

\section{DISCUSSION}

The aim of the present study was to validate the K-DMSS in a sample of elderly patients with delirium admitted to a general hospital.

There have been numerous attempts to assess the motor symptoms of delirium with psychomotor checklists. ${ }^{911}$ Previously, authors have used several delirium severity scales, including the Delirium Rating Scale (DRS), ${ }^{23}$ DRS-R98, ${ }^{24} \mathrm{Me}-$ morial Delirium Assessment Scale (MDAS), ${ }^{2,25}$ and Richmond Agitation/Sedation Scale. ${ }^{26}$ In some cases, clinical observation has been used to identify the delirium subtype. ${ }^{27}$ However, reported findings have been heterogeneous because of the lack of a standard method with a clear cutoff value and the use of 


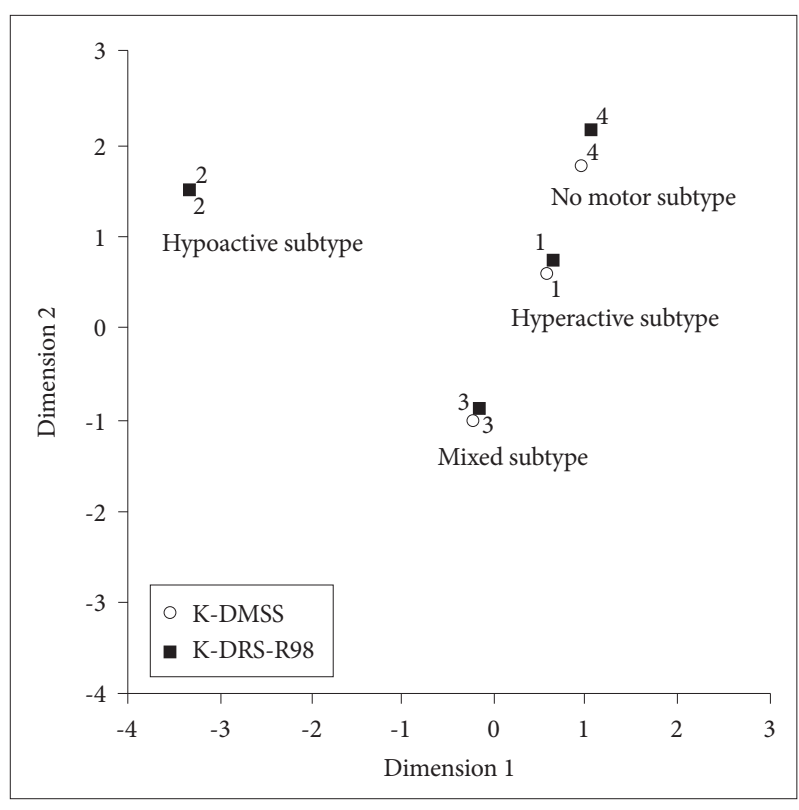

Figure 2. Correspondence analysis-relationship between K-DMSS and K-DRS-R98.

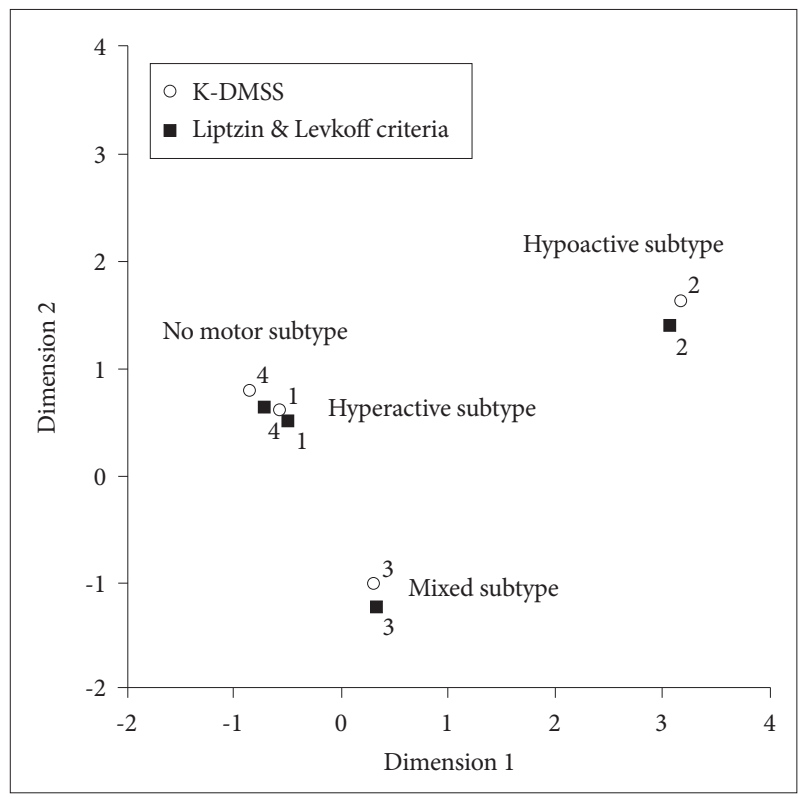

Figure 3. Correspondence analysis-relationship between K-DMSS and Liptzin \& Levkoff criteria.

instruments assessing non-motor symptoms. Meagher et al. compared the identification of delirium subtypes with various common methods ${ }^{9-11}$ and the DRS-R98, ${ }^{13}$ finding just 34\% concordance. ${ }^{14}$ Subsequently, they used items from these various assessments and combined them to create the DMC. From 30 items, they selected 11, that were specific to patients with delirium, and investigated correlation between them and motor items of the DRS-R98. This process created a new assessment, the DMSS. ${ }^{15}$ The latter allows more precise detection of motor subtypes than other methods by purely focusing on motor symptoms. In addition, it is a simple and easy scale that can be used by non-medical staff, and has been demonstrated to have concurrent and predictive validity. ${ }^{15,16,28}$

More recently, the amended DMSS was developed by adding "increased speed of actions" to hyperactive items, and "decreased volume of speech" to hypoactive items, following investigation of hospitalized patients referred to consultationliaison psychiatry. ${ }^{29}$ Later, an abbreviated version of the DMSS $(\text { DMSS- } 4)^{30}$ was presented for brief use in clinical settings where time constraints were present, and this was highly concordant with the original DMSS.

In terms of reliability, our results show that Cronbach's alpha coefficient for items included in the K-DMSS was 0.79, indicating good internal inconsistency. Furthermore, Cohen's kappa coefficient was 0.86 , indicating good inter-rater reliability. Therefore, we demonstrated that the K-DMSS is reliable. Indeed, our results suggest slightly higher reliability than those reported in a validation study by Slor et al. ${ }^{31}$ in which Cronbach's alpha was 0.72 .

To evaluate validity, we classified delirium motor subtypes with the K-DRS-R98 and Liptzin \& Levkoff criteria, because both have been commonly used in previous studies. Cohen's kappa coefficient for the motor subtypes was 0.81 between the K-DMSS and K-DRS-R98, and 0.78 between the K-DMSS and Liptzin and Levkoff criteria. Therefore, our results demonstrate concurrent validity between the K-DMSS and previously used measure. Validity was also higher than that reported in by Slor et al. ${ }^{31}$ in which Cohen's kappa coefficient was 0.73 between the DMSS and the DRS-R98. Regarding sensitivity and specificity, except for the sensitivity value (0.5) of the no motor subtype between K-DMSS and Liptzin \& Levkoff criteria, the values ranged from approximately 0.80 to 1 (Tables 4 and 6), thereby yielding high accuracy of K-DMSS in classifying each motor subtype and comparing with previously used measures.

In addition, correspondence analysis was used to illustrate the correlations of each subtype with one another, as classified by each scale. In the graphical representation (Figure 2), the distance between each point represents the accordance of each subtype. Each subtype was visualized in the adjacent area. Therefore, the motor subtypes classified with the K-DMSS were concordant with those classified with the other methods.

According to the K-DMSS, the hyperactive subtype was the most common in the present study, affecting $50 \%$ of the sample. This is consistent with the results of Grover et al. ${ }^{32}$ who reported that the hyperactive subtype was most common at $50.15 \%$, but not with the findings of Meagher et al. ${ }^{15}$ or Uchida et al. ${ }^{33}$ who reported that the hypoactive subtype was most common, affecting $35 \%$ and $58 \%$ of their samples, respectively. Grover et al..$^{32}$ studied hospitalized patients who had been ad- 
mitted for various diseases and subsequently referred to consultant-liaison psychiatry as a result of delirium. In contrast, Meagher et al. ${ }^{15}$ and Uchida et al..$^{33}$ investigated patients who received palliative care or those with advanced cancer, who subsequently presented with symptoms of delirium. Such inconsistent findings may suggest that motor subtype classification may depend on the population characteristics.

In the present study, the hypoactive subtype was relatively less common than the hyperactive and mixed subtypes using all the three assessment methods. This subtype will less likely attract the attention of a caregiver or nurse because there will be less or no overt behavioral disturbance in patients with the hypoactive subtype. ${ }^{34}$ In contrast, behavioral disturbances in the hyperactive subtype are obvious. As such, some patients with the hypoactive subtype may not have been referred for psychiatric care.

There are several limitations to the present study, which should be borne in mind when considering our results. First, we assessed patients with altered mental state, referred to the psychiatric consultation liaison service. As we did not objectively evaluate the previous mental state of patients and excluded patients with dementia on the basis of the information provided by caregivers, the association between delirium and dementia was not considered. Furthermore, as mentioned above, hyperactive symptoms may have been more detectable; however, hypoactive symptoms may not have been recognized as characteristic of delirium by physicians. This may have resulted in a selection bias. Further studies assessing patients from the time of admission may overcome these limitations. Second, the potential influence of prescribed medications, such as benzodiazepine, antipsychotics, anticholinergics, and opiates, during the assessment was not considered. Third, the scale required symptoms to be rated on the basis of behavior $24 \mathrm{~h}$ before the assessment. Nonetheless, it is likely that information provided by patients or caregivers may have referred to times before the previous $24 \mathrm{~h}$. Finally, the sample size was small, and inter-rater reliability was only assessed in a subset of patients.

Nonetheless, to our knowledge, this is the first validation study of the DMSS in elderly Korean patients. Further work in various populations to determine the efficacy of the scale will contribute to our understanding of delirium and its detection, treatment, and prognosis. Furthermore, additional research is required using the various versions of the DMSS, which are continually revised.

\section{REFERENCES}

1. Meagher DJ, O'Hanlon D, O’Mahony E, Casey PR, Trzepacz PT. Relationship between symptoms and motoric subtype of delirium. J Neuropsychiatry Clin Neurosci 2000;12:51-56.
2. Morita T, Tei Y, Tsunoda J, Inoue S, Chihara S. Underlying pathologies and their associations with clinical features in terminal delirium of cancer patients. J Pain Symptom Manage 2001;22:997-1006.

3. Balan S, Leibovitz A, Zila SO, Ruth M, Chana W, Yassica B, et al. The relation between the clinical subtypes of delirium and the urinary level of 6-SMT. J Neuropsychiatry Clin Neurosci 2003;15:363-366.

4. Marcantonio E, Ta T, Duthie E, Resnick NM. Delirium severity and psychomotor types: their relationship with outcomes after hip fracture repair. J Am Geriatr Soc 2002;50:850-857.

5. Meagher D. Motor subtypes of delirium: past, present and future. Int Rev Psychiatry 2009;21:59-73.

6. Meagher D, Leonard M. The active management of delirium: improving detection and treatment. Adv Psychiatry Treat 2008;14:292-301.

7. Lipowski ZJ. Delirium: Acute Confusional States. New York: Oxford University Press; 1990.

8. Lipowski Z. Transient Cognitive Disorders (Delirium, Acute Confusional Sates) in the Elderly. In: Lipowski Z, Editor. Psychosomatic Medicine and Liaison Psychiatry. Boston, MA: Springer, 1983, p.289306.

9. Lipowski ZJ. Delirium in the elderly patient. New Eng J Med 1989;320: 578-582.

10. Liptzin B, Levkoff SE. An empirical study of delirium subtypes. Br J Psychiatry 1992;161:843-845.

11. O'Keeffe ST, Lavan JN. Clinical significance of delirium subtypes in older people. Age Ageing 1999;28:115-119.

12. de Rooij SE, van Munster BC, Korevaar JC, Casteelen G, Schuurmans MJ, van der Mast RC, et al. Delirium subtype identification and the validation of the Delirium Rating Scale-Revised-98 (Dutch version) in hospitalized elderly patients. Int J Geriatr Psychiatry 2006;21:876-882.

13. Trzepacz PT, Mittal D, Torres R, Kanary K, Norton J, Jimerson N. Validation of the Delirium Rating Scale-revised-98: comparison with the delirium rating scale and the cognitive test for delirium. J Neuropsychiatry Clin Neurosci 2001;13:229-242.

14. Meagher DJ, Moran M, Raju B, Gibbons D, Donnelly S, Saunders J, et al. Motor symptoms in 100 patients with delirium versus control subjects: comparison of subtyping methods. Psychosomatics 2008;49:300-308.

15. Meagher D, Moran M, Raju B, Leonard M, Donnelly S, Saunders J, et al. A new data-based motor subtype schema for delirium. J Neuropsychiatr Clin Neurosci 2008;20:185-193.

16. Godfrey A, Leonard M, Donnelly S, Conroy M, ÓLaighin G, Meagher D. Validating a new clinical subtyping scheme for delirium with electronic motion analysis. Psychiatry Res 2010;178:186-190.

17. Lee J, Lee H, Kim S, Kim J. A study for the diagnosis, severity and subtypes of delirium using the Korean version of Delirium Rating ScaleRevised-98 (K-DRS-98). J Korean Soc Biol Ther Psychiatry 2008;14:101109.

18. Association AP. Diagnostic and Statistical Manual of Mental Disorders (DSM-5®). Washington DC: American Psychiatric Pub; 2013.

19. Lim KO, Kim SY, Lee YH, Lee SW, Kim JL. A validation study for the Korean version of Delirium Rating Scale-Revised-98 (K-DRS-98). J Korean Neuropsychiatr Assoc 2006;45:518-526.

20. Albert MS, Levkoff SE, Reilly C, Liptzin B, Pilgrim D, Cleary PD, et al. The delirium symptom interview: an interview for the detection of delirium symptoms in hospitalized patients. J Geriatr Psychiatry Neurol 1992;5:14-21.

21. Landis JR, Koch GG. The measurement of observer agreement for categorical data. Biometrics 1977:159-174.

22. Trzepacz PT, Meagher DJ, Wise M. Neuropsychiatric Aspects of Delirium. In: Yudofsky SC, Hales RE, Editors. Textbook of Neuropsychiatry 5th ed. Washington, DC: American Psychiatric Press; 2008. p.445-518.

23. Platt MM, Breitbart W, Smith M, Marotta R, Weisman H, Jacobsen P. Efficacy of neuroleptics for hypoactive delirium. J Neuropsychiatry Clin Neurosci 1994;6:66-67.

24. Gupta A, Saravay S, Trzepacz P Chirayu P. Delirium Motoric Subtypes. Psychosomatics 2005;46:158. 
25. Breitbart W, Tremblay A, Gibson C. An open trial of olanzapine for the treatment of delirium in hospitalized cancer patients. Psychosomatics 2002;43:175-182.

26. Peterson J, Truman B, Shintani A, Thomason J, Jackson J, Ely E. The prevalence of delirium subtypes in medical icu patients. J Am Geriatr Soc 2003;51:S174.

27. Olofsson SM, Weitzner MA, Valentine AD, Baile WF, Meyers CA. A retrospective study of the psychiatric management and outcome of delirium in the cancer patient. Support Care Cancer 1996;4:351-357.

28. Meagher DJ, Leonard M, Donnelly S, Conroy M, Adamis D, Trzepacz PT. A longitudinal study of motor subtypes in delirium: relationship with other phenomenology, etiology, medication exposure and prognosis. J Psychosom Res 2011;71:395-403.

29. Grover S, Mattoo SK, Aarya KR, Das PP, Chakrabarty K, Trzepacz P, et al. Replication analysis for composition of the Delirium Motor Subtype Scale (DMSS) in a referral cohort from Northern India. Psychiatry Res 2013;206:68-74.
30. Meagher D, Adamis D, Leonard M, Trzepacz P, Grover S, Jabbar F, et al. Development of an abbreviated version of the Delirium Motor Subtyping Scale (DMSS-4). Int Psychogeriatr 2014;26:693-702.

31. Slor CJ, Adamis D, Jansen RW, Meagher DJ, Witlox J, Houdijk AP, et al. Validation and psychometric properties of the Delirium Motor Subtype Scale in elderly hip fracture patients (Dutch version). Arch Gerontol Geriatr 2014;58:140-144.

32. Grover S, Sharma A, Aggarwal M, Mattoo SK, Chakrabarti S, Malhotra $\mathrm{S}$, et al. Comparison of symptoms of delirium across various motoric subtypes. Psychiatry Clin Neurosci 2014;68:283-291.

33. Uchida M, Okuyama T, Ito Y, Nakaguchi T, Miyazaki M, Sakamoto M, et al. Prevalence, course and factors associated with delirium in elderly patients with advanced cancer: a longitudinal observational study. Jpn J Clin Oncol 2015;45:934-940.

34. Inouye SK, Foreman MD, Mion LC, Katz KH, Cooney LM Jr. Nurses' recognition of delirium and its symptoms: comparison of nurse and researcher ratings. Arch Intern Med 2001;161:2467-2473. 